\title{
FKBP5 DNA methylation does not mediate the association between childhood maltreatment and depression symptom severity in the Detroit Neighborhood Health Study
}

\author{
Angela C. Bustamante ${ }^{a}$, Allison E. Aiello ${ }^{b}$, Guia Guffanti ${ }^{c}$, Sandro Galea ${ }^{d}$, \\ Derek E. Wildman ${ }^{\mathrm{e}, \mathrm{f}}$, Monica Uddin ${ }^{\mathrm{a}, \mathrm{f}, \mathrm{g}, \text { * }}$ \\ ${ }^{a}$ Neuroscience Program, University of Illinois at Urbana-Champaign, Urbana, IL, USA \\ ${ }^{\mathrm{b}}$ Department of Epidemiology, University of North Carolina Gillings School of Global Public Health, USA \\ ${ }^{c}$ Department of Psychiatry, McLean Hospital, Harvard Medical School, Belmont, MA, USA \\ ${ }^{\mathrm{d}}$ Boston University School of Public Health, Boston, MA, USA \\ e Department of Molecular and Integrative Physiology, University of Illinois at Urbana-Champaign, Urbana, IL, USA \\ ${ }^{\mathrm{f}}$ Carl R. Woese Institute for Genomic Biology, University of Illinois at Urbana-Champaign, Champaign, IL, USA \\ ${ }^{g}$ Department of Psychology, University of Illinois at Urbana-Champaign, Champaign, IL, USA
}

\section{A R T I C L E I N F O}

\section{Article history:}

Received 16 March 2017

Received in revised form

8 September 2017

Accepted 14 September 2017

\section{Keywords:}

Epigenetics

Gene expression

Childhood maltreatment

Major depressive disorder

Mediation

\begin{abstract}
A B S T R A C T
Exposure to childhood maltreatment increases the risk of developing mental illness later in life. Childhood maltreatment and depression have both been associated with dysregulation of the hypothalamicpituitary-adrenal (HPA) axis-a key regulator of the body's stress response. Additionally, HPA axis dysregulation has been implicated in the etiology of a range of mental illnesses. A substantial body of work has shown history of childhood maltreatment alters DNA methylation levels within key HPA axis genes. We therefore investigated whether one of these key genes, FKBP5 mediates the relationship between childhood maltreatment and depression, and assessed FKBP5 DNA methylation and gene expression within 112 adults from the Detroit Neighborhood Health Study (DNHS). DNA methylation was assessed in 4 regions, including the upstream promoter, downstream promoter, and two glucocorticoid response elements (GREs) via pyrosequencing using whole blood derived DNA; Taqman assays measured relative RNA expression from leukocytes. Mediation analyses were conducted using sequential linear regression. Childhood maltreatment was significantly associated with depression symptom severity (FDR $<0.006$ ), but was not a significant predictor of DNA methylation in any of the four loci examined. FKBP5 showed elevated expression levels in participants with vs. without a history of depression ( $p<0.001)$; no significant difference in gene expression levels was observed in relation to childhood maltreatment ( $p>0.05$ ). Our results suggest DNA methylation does not mediate the childhood maltreatmentdepression association in the DNHS.
\end{abstract}

\section{Introduction}

Adverse early life experiences, such as childhood maltreatment (CM), increase susceptibility for developing depression (Hussey et al., 2006; Nanni et al., 2012; Nelson et al., 2017), posttraumatic stress disorder (PTSD) (Stein et al., 1996), bipolar

\footnotetext{
* Corresponding author. Department of Psychology, Carl R. Woese Institute for Genomic Biology, University of Illinois at Urbana-Champaign, 603 E. Daniel St, 527 Psychology, Champaign, IL 61820, USA

E-mail address: muddin@illinois.edu (M. Uddin).
}

disorder (Afifi et al., 2008), and other mental health disorders (MacMillan et al., 2001; Martin-Blanco et al., 2014; McGowan et al., 2009 ) in adulthood. In addition, exposure to CM has been shown to dysregulate the hypothalamic-pituitary-adrenal (HPA) axis, a master regulator of the body's stress response (reviewed in (Van Voorhees and Scarpa, 2004)). Substantial work has demonstrated that dysregulation of the HPA axis increases the likelihood of developing mood and anxiety disorders (Appel et al., 2011; Ceulemans et al., 2011; Espejo et al., 2007; Guerry and Hastings, 2011; Nemeroff, 2004). Moreover, it has been well-documented that exposures during early life, including CM, impact DNA methylation $(5 \mathrm{mC})$ levels within genes of the HPA axis (Martin- 
Blanco et al., 2014; McGowan et al., 2009; Romens et al., 2015; van der Knaap et al., 2014) making the HPA axis a logical starting point for studies examining the epigenetic impact of $\mathrm{CM}$.

FK506 Binding Protein 5 (FKBP5) is a gene whose product is involved in HPA axis function by negatively regulating the stress response. The HPA axis is activated by stress and is regulated by two levels of negative feedback: long range and ultra-short intracellular feedback loops. Long range feedback occurs when high levels of cortisol in the blood stream are detected by the hypothalamus, resulting in decreased excretion of corticotropin releasing hormone (CRH) and subsequent tamping down of the stress response (Binder, 2009; Binder et al., 2008; Davies et al., 2002; Yang et al., 2012). Ultra-short feedback occurs at the cellular level and involves FKBP5, which acts as a negative regulator of the glucocorticoid receptor (GR) (Appel et al., 2011; Binder, 2009; Binder et al., 2008; Yang et al., 2012). The presence of FKBP5, when bound to a multi-protein chaperone complex in the cytoplasm, lowers the binding affinity between cortisol and the GR (Binder, 2009). When cortisol is able to successfully bind to the GR, the GR-cortisol complex translocates into the nucleus, where it binds to glucocorticoid response elements (GREs) (Binder et al., 2008; Yang et al., 2012) and acts as a transcription factor to upregulate FKBP5 transcription. Increased expression of FKBP5 reduces GR sensitivity, leading to more unbound cortisol in the cytoplasm and in the blood, thereby activating the long feedback loop (Binder, 2009).

Despite FKBP5's prominent role in the regulation of the stress response, relatively few studies have examined the relationship between CM and FKBP5 5mC in humans. Klengel et al. (2013). examined FKBP5 $5 \mathrm{mC}$ in participants with a history of childhood trauma compared to a control group. They reported significantly decreased $5 \mathrm{mC}$ at several glucocorticoid response elements (GREs) and a significant interaction between childhood trauma exposure and genetic variation at rs1360780 to predict $5 \mathrm{mC}$ at intron 7 (Klengel et al., 2013). A second study, by Tyrka et al. examined the association of CM and FKBP5 $5 \mathrm{mC}$ at intron 7 in a cohort of preschool aged children, and found significantly decreased FKBP5 $5 \mathrm{mC}$ of two CpG sites within intron 7 and found no significant interaction between SNP rs1360780 and lifetime contextual stress on FKBP5 5mC of intron 7 (Tyrka et al., 2015). A third study in children, by Weder et al. employed a genome-wide approach using Illumina's $450 \mathrm{~K}$ Bead Chip to examine $5 \mathrm{mC}$ and more broadly the relationship between CM and depression (Weder et al., 2014). They reported significant differences among $\mathrm{CM}$ cases compared to controls at several CpG sites within FKBP5. However, due to the incomplete coverage of the FKBP5 region by the $450 \mathrm{~K}$ array, the region examined in the two earlier studies was not assessed.

Two additional groups have examined FKBP5 $5 \mathrm{mC}$ in relation to other stressful life events. Hönhe et al. examined the impact of psychosocial stress on FKBP5 $5 \mathrm{mC}$ and gene expression (GE) within the context of major depression history and FKBP5 genotype. They found a significant interaction of SNP genotype at rs1360780 and depression status on FKBP5 $5 \mathrm{mC}$ of intron 7, however this finding did not survive multiple test corrections (Hohne et al., 2015). Lastly, Non et al. found the length of time in institutionalized care was significantly negatively correlated with FKBP5 intron 7 CpG site 1 $5 \mathrm{mC}$ after correcting for multiple hypothesis testing (Non et al., 2016).

These studies reported significantly different FKBP5 $5 \mathrm{mC}$ associated with either exposure to CM or major depression, however none of them directly examined the relationship among $\mathrm{CM}, 5 \mathrm{mC}$, and depression - yet, given the demonstrated relationship between $\mathrm{CM}$ and $5 \mathrm{mC}$, as well as differential $5 \mathrm{mC}$ in relation to depression in FKBP5 (Hohne et al., 2015) and other HPA axis genes (Na et al., 2014; Nantharat et al., 2015; Tyrka et al., 2012), a plausible relation exists among these factors. To address this possibility, we examined whether FKBP5 5mC mediates the relationship between $\mathrm{CM}$ and depression, and if the altered $5 \mathrm{mC}$ subsequently influences GE levels of FKBP5. Our primary analyses focused on the introns implicated in previous work as being influenced by CM (Klengel et al., 2013; Tyrka et al., 2015), while the FKBP5 promoter region analyses reflect a smaller exploratory dataset that tested more canonical influences of $5 \mathrm{mC}$ on GE.

\section{Methods}

\subsection{Participant selection}

The Detroit Neighborhood Health Study (DNHS) was approved by the institutional review board at the University of Michigan and the University of North Carolina at Chapel Hill. Participants were selected from the DNHS, a longitudinal, population-based representative sample of adult residents from Detroit, MI (Goldmann et al., 2011; Uddin et al., 2010). All participants provided informed consent prior to participation. Selection for inclusion in this study was based on the availability of whole-blood derived DNA, leukocyte-derived RNA, and complete survey data regarding $\mathrm{CM}$ and depression histories. The full sample $(\mathrm{N}=112)$ was tested for intron 2 and intron 7 , and a subset of participants $(\mathrm{N}=72)$ was selected for the promoter region assays.

\subsection{Childhood maltreatment}

CM history was collected via structured telephone interviews assessing the severity, duration, and frequency of each event type. Assessment of CM was based on an adaptation of the Conflict Tactics Scale (CTS) (Straus, 1979) and the Childhood Trauma Questionnaire (CTQ) (Bernstein et al., 1997), as previously described (Keyes et al., 2012; Uddin et al., 2013). CTS items assessed physical and emotional abuse before age 11 , with responses rated on a 5 point scale. CTQ assessed physical and sexual abuse before age 18 , rating responses on a 3-point scale. In this study, a binary variable of $\mathrm{CM}$ exposure was used, such that $\mathrm{CM}$ exposure $(\mathrm{N}=56)$ was defined as any individual belonging to the upper quartile for $\mathrm{CM}$ score within the full wave 2 DNHS survey sample $(\mathrm{N}=1588)$. Participants without exposure to $\mathrm{CM}(\mathrm{N}=56)$ belonged to the bottom quartile of CM score within the full wave 2 DNHS survey sample.

\subsection{Depression measure}

Depression symptom severity (depSS) scores were based on participant responses from the Patient Health Questionnaire (PHQ9) (Kroenke et al., 2001), rated on a 4-point scale for each of the 9 questions with each question ranging from 0 (not at all) to 3 (nearly every day); and total depression severity scores ranging from 0 to 27 as described in (Uddin et al., 2013). Lifetime major depressive disorder (MDD) was assessed at each wave of the DNHS using the PHQ-9, with additional questions to assess the timing and duration of symptoms, consistent with DSM-IV criteria (American Psychiatric, 1994) and previously validated (Uddin et al., 2011). Participant depression information was taken from the same wave as the participants' biospecimens tested in the experiments described below. In this study, the continuous measure of depSS was used in the regression models. Within the GE analyses MDD was defined as history of lifetime MDD.

\subsection{Antidepressant medication}

Participant medication information was obtained during the inhome visit at the time of biologic sample collection and has been 
described previously (Bustamante et al., 2016) and is included in the regressions as a covariate.

\subsection{Genotype}

Participant genotype of FKBP5 SNP rs1360780 was imputed from data collected by the Illumina HumanOmniExpress BeadChip. PLINK (Purcell et al., 2007) was used to impute the genotype of rs1360780 for the DNHS participants. A subset of the study participants $(\mathrm{N}=100)$ had available genotype information.

\subsection{Sample preparation}

\subsubsection{DNA and RNA collection}

Whole blood was collected via venipuncture from study participants during scheduled in-home visits by a phlebotomist. DNA was isolated from whole blood and concentration was verified via Nanodrop 1000 (Thermofisher Scientific, Waltham, MA) as previously described (Bustamante et al., 2016). RNA was isolated from leukocytes and subjected to the following criteria to ensure high quality RNA was obtained, including a RNA integrity number (RIN) $\geq 5,28 \mathrm{~s} / 18 \mathrm{~s} \geq 1.0$, and $260 / 280 \geq 1.7$ (Fleige and Pfaffl, 2006; Fleige et al., 2006). RNA sample RIN values and $28 \mathrm{~s} / 18 \mathrm{~s}$ ratios were calculated using the 2100 Bioanalyzer (Agilent, Wilmington, DE) to determine RNA quality.

\subsubsection{Peripheral blood mononuclear cell counts}

At the time of blood draw, two FICOLL gradient containing $8 \mathrm{ml}$ BD Vacutainers CPT ${ }^{\mathrm{TM}}$ with sodium citrate (Franklin Lakes, $\mathrm{NJ}$ ) were used for the collection of peripheral blood mononuclear cell (PBMC) samples and processed as previously described (Bustamante et al., 2016).

\subsubsection{Bisulfite conversion}

Using Qiagen's Epitect Bisulfite Kit, 750 ng of participant DNA was bisulfite converted following the manufacturer's recommended protocol. Negative controls containing RNAse/DNAse free water in place of DNA were included with each bisulfite conversion. High and low methylation control DNA purchased from Zymo Research (Irvine, CA) was bisulfite converted along with the participant samples in order to assess assay performance.

\subsection{PCR amplification \& pyrosequencing}

$5 \mathrm{mC}$ upstream of the transcription start site (TSS) (CHR6:35,656,797-35,656,920), downstream of the TSS (CHR6:35,655,829-35,655,843), and introns 2 (CHR6:35,558,310 $-35,558,785)$ and 7 (CHR6:35,607,754-35,608,049) of FKBP5 were assessed via pyrosequencing. These regions were targeted in part based on: (i) previous work within FKBP5 (intron 2 and 7 (Klengel et al., 2013):), and (ii) previous work demonstrating an association between early life adversity and $5 \mathrm{mC}$ in promoter regions of related HPA axis genes (i.e. NR3C1 (Oberlander et al., 2008; Tyrka et al., 2012)). All assay files were designed and created using the PyroMark Q24 Assay Design Software 2.0 (Qiagen). 5mC of FKBP5's Intron 2 and 7, which encompass GREs, has been previously investigated by (Klengel et al., 2013; Tyrka et al., 2015; Yehuda et al., 2015a) (Fig. 1). PCR and pyrosequencing primers for Intron 2 and 7 have been previously described in (Klengel et al., 2013). Due to the read length of the assay associated with the intron 7 primers developed by Klengel et al. intron 7 CpG 3 methylation data was not reliably obtained; therefore, a new sequencing primer for Intron 7 CpG 3 was designed and used with the amplicon amplified by the PCR primers as described by (Klengel et al., 2013). The two intron 2 $\mathrm{CpG}$ sites are both located near a consensus GRE sequence, $\sim 552 \mathrm{bp}$ away. Within intron $7 \mathrm{CpG}$ sites 1 and 3 surround the consensus GRE sequence: $\mathrm{CpG} 1$ is $15 \mathrm{bp}$ away from the closest GRE consensus sequence, and CpG 3 is 47bp away from the same GRE consensus sequence; in contrast, CPG 2 is located within the consensus GRE sequence. Within the promoter region, two separate loci were examined. The first was a ready-made Qiagen assay targeting $3 \mathrm{CpG}$ sites within intron 1 and overlapped a CpG island TSS, denoted as downstream promoter (Fig. 1). The second was newly developed using the PyroMark Q24 Assay Design Software 2.0 (Qiagen). The newly developed primer set encompassed $11 \mathrm{CpG}$ sites within several transcription factor binding sites (TFBS) including EGR-1, and overlapped a CpG island upstream of the TSS, denoted as upstream promoter (Fig. 1).

All PCR and sequencing primers were validated according to the recommendations in the PyroMark manual to ensure high quality primers were used. Additionally, each primer set was tested using commercially available Human high and low methylation control DNA (Zymo Research) that was bisulfite converted on site. For each locus examined, the high methylation control DNA averages and ranges were: $91.1 \%$ for the upstream promoter with methylation ranging from $74 \%$ to $100 \%$; $96.7 \%$ for the downstream promoter ranging from $92 \%$ to $100 \%$; $76.1 \%$ for intron 2 , ranging from $67 \%$ to $85 \%$; and $96.4 \%$ for intron 7 ranging from $93 \%$ to $100 \%$. The low methylation control DNA averages and ranges were: $8 \%$ for the upstream promoter, with methylation ranging from $2 \%$ to $32 \% ; 6.6 \%$ for the downstream promoter ranging from $5 \%$ to $8 \% ; 27.9 \%$ for intron 2, ranging from $27 \%$ to $30 \%$ intron 2; and $60.6 \%$ for intron 7 ranging from $54 \%$ to $68 \%$. Bisulfite converted participant DNA was amplified using Qiagen's PyroMark PCR kit for all four loci of FKBP5 examined in this study. PCRs were run in duplicate and contained 20 ng of bisulfite converted participant DNA as starting template. No template controls were run in duplicate and were included with each set of PCRs as a negative control as they did not contain any DNA. Qiagen's PyroMark Q24 Pyrosequencer was used to detect $5 \mathrm{mC}$ levels following manufacturer's protocols and default settings.

\section{Upstream Promoter Assays:}

Upstream Promoter Primerset_1

Forward PCR primer (biotinylated): 5'-AGGGTTTAGGGGTTGTTAGT-3'

Reverse PCR primer: 5'-TCCACCATCCCTTCTCTATAAC-3' Sequencing primer: 5'-ATAATAAAAACCAAACCTCATTTAC$3^{\prime}$

Annealing temperature: $58{ }^{\circ} \mathrm{C}$

Targets $9 \mathrm{CpG}$ sites

Upstream Promoter Primerset_2

Forward PCR primer: 5'-AGGGTTTAGGGGTTGTTAGT-3'

Reverse PCR primer (biotinylated): 5'-TTTCCACCATCCCTTCTCTATAAC-3'

Sequencing primer: $5^{\prime}$-GTTTATGTAAATGAGGT-3'

Annealing temperature: $58{ }^{\circ} \mathrm{C}$

Targets 2 CpG sites

Downstream Promoter Assay:

Assay Name: Hs_FKBP5_01_PM PyroMark CpG assay

Qiagen Catalogue Number: PM00120946

Sequencing primer: $5^{\prime}$ - CGTTCAGCCGCTTCGG - $3^{\prime}$

Annealing temperature: $56{ }^{\circ} \mathrm{C}$

Targets $3 \mathrm{CpG}$ sites

Intron 7 Assay:

FKBP5 Intron 7 CpG 3 sequencing primer: 5'- AAGTTGTATTTTATTTTTTTAAGGA - $3^{\prime}$

Annealing temperature: $57^{\circ} \mathrm{C}$

Targets $1 \mathrm{CpG}$ site 


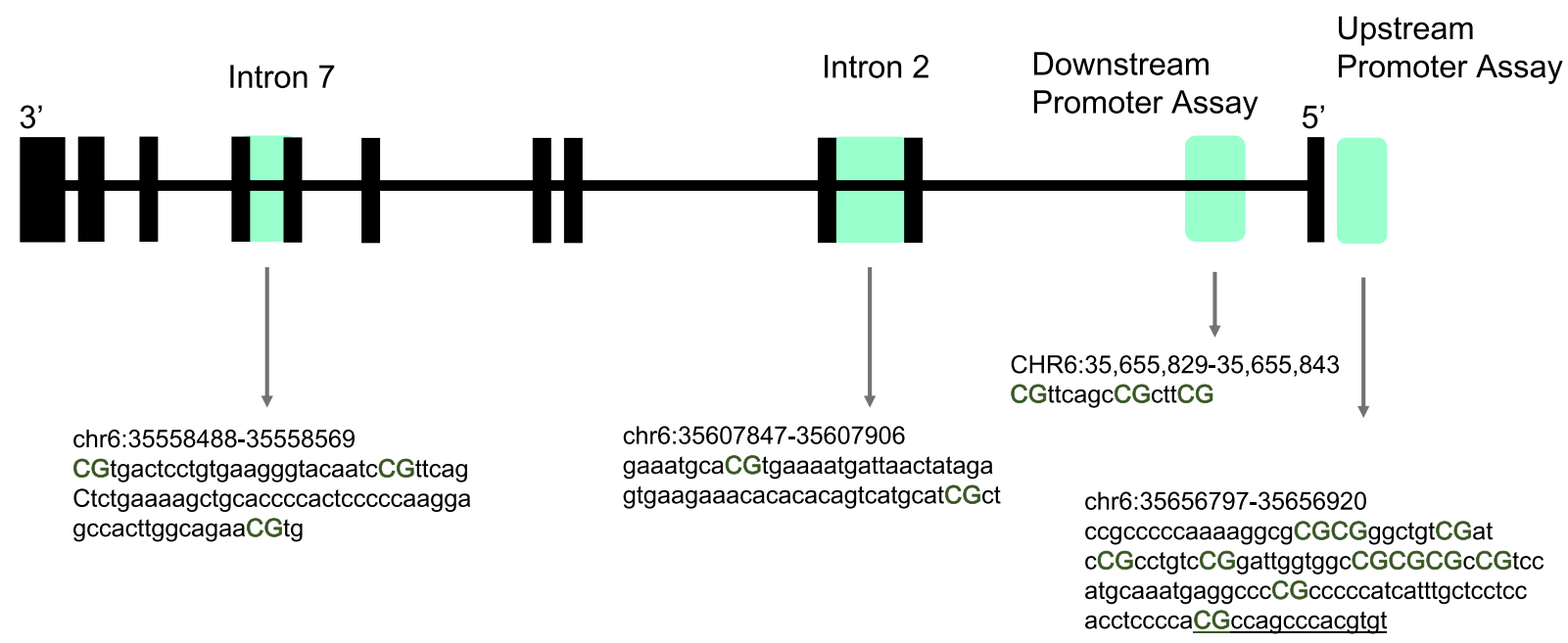

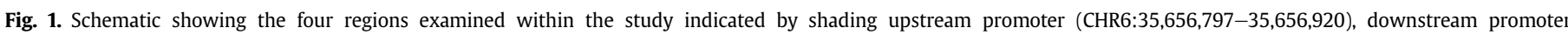

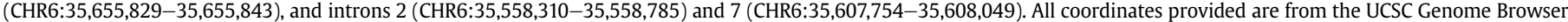

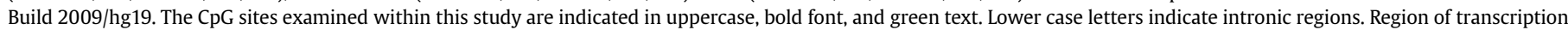

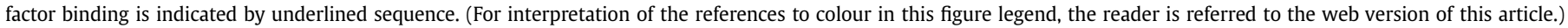

\section{PCR Program: (same for all primer sets)}

\section{Initial}

15 minutes $95^{\circ} \mathrm{C}$

Denaturation

Annealing

Extension

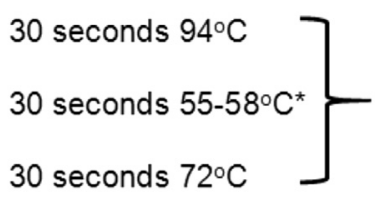

50 cycles

Final

10 minutes $72^{\circ} \mathrm{C}$

Hold

$4^{\circ} \mathrm{C}$

${ }^{*}$ Annealing temperature varies for each of the PCR primers.

\subsection{Reverse transcription \& real-time $P C R$}

FKBP5 mRNA expression levels were assessed in participants with available total RNA $(\mathrm{N}=71)$. Total RNA was reverse transcribed into cDNA following the manufacturer's protocol using the High Capacity Reverse Transcription Kit purchased from Applied BioSystems (Foster City, CA). Ready-made Taqman GE assays (Applied Biosystems) were used to measure relative transcript levels of the target gene FKBP5 (Hs015610061_m1) and the control gene TATA Binding Protein, TBP (Hs00427621_m1) run in separate wells. Reactions were performed in triplicate for each participant. Each reaction was $20 \mu \mathrm{l}$ and contained $10 \mathrm{ng}$ of participant cDNA for both the test and control genes. Reactions were run on the HT7500 Fast Real-Time PCR machine (Applied BioSystems, Foster City, CA) following the manufacturer's recommended protocol.

\subsection{Pyrosequencing data analysis}

$5 \mathrm{mC}$ levels were averaged across $\mathrm{CpG}$ sites within each of the four tested loci (upstream promoter, downstream promoter, intron 2 , and intron 7). This step was performed based on the assumption that $\mathrm{CpG}$ sites within a functional region, such as the promoter region, a TFBS, or a GRE are correlated, based on previous empirical evidence (Jones, 2012). Additionally, the composition of CpG sites within intron 2 and 7 that were averaged correspond to the CpGs included in the bins as reported by (Klengel et al., 2013). The distribution of averaged $5 \mathrm{mC}$ data obtained for each region was evaluated using boxplots, histograms, and tested for normality using the Shapiro-Wilk test of normality in IBM SPSS Statistics for Windows, version 23.0 (IBM Corp., Armonk, NY).

\subsubsection{Demographic statistics}

Independent samples t-tests were used to test for bivariate associations between CM exposure and demographic covariates, as well as depression and demographic covariates. Chi-square tests were used to test for associations between $\mathrm{CM}$ exposure and the demographic variables of sex, race, and genotype. Similarly, Chisquare tests were used to test for the association between depression history and sex, race, and genotype. All statistical tests were two-tailed and results were considered significant when $\mathrm{p}<0.05$. Tests were conducted using SPSS version 23.0.

\subsubsection{Mediation analyses}

$5 \mathrm{mC}, \mathrm{CM}$, and depSS information for each participant was used to carry out mediation analyses using linear regressions (Fig. 2). The analysis was performed using SPSS version 23.0, following criteria outlined in (Baron and Kenny, 1986). Specifically, we assessed whether: (a) CM is associated with depSS after controlling for confounders (i.e. age, sex, race, PBMC count, and antidepressant medication use); (b) CM is associated with FKBP5 $5 \mathrm{mC}$ levels, after

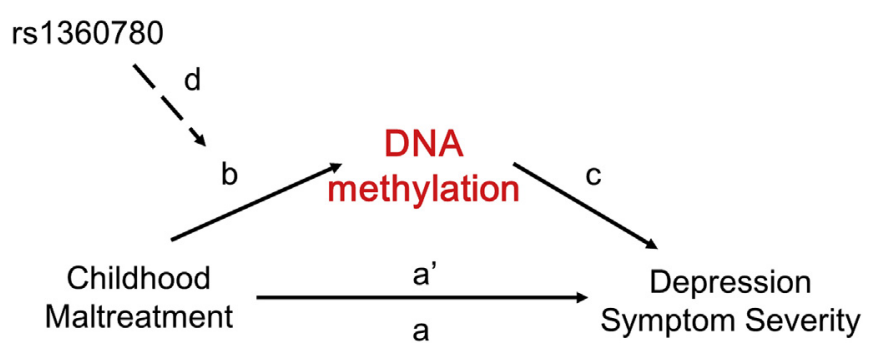

Fig. 2. Schematic showing the mediation framework for our analysis. Letters indicate the order in which the regression models were tested. Note analysis "d" was only tested for Intron 7. 
controlling for confounders; (c) if FKBP5 $5 \mathrm{mC}$ is a predictor of depSS, after controlling for confounders; and lastly, if a, b and c were significant, we tested (a'), i.e. if the effect of CM on depSS at FKBP5 regions is attenuated after controlling for $5 \mathrm{mC}$ at those regions, as well as other confounders. All regression models included age, sex, self-reported race, PBMC count, and antidepressant medication information as covariates. A secondary analysis tested the potential interaction between CM and FKBP5 SNP rs1360780 within intron 7, as previously reported in (Klengel et al., 2013; Tyrka et al., 2015), in a subset of individuals with available genotype data $(n=100)$. For the model testing for an interaction, the main effect model was run first, followed by the interaction model. To address the concern of multiple hypothesis testing and potential Type I errors, the false discovery rate (FDR) was calculated as outlined in (Benjamini et al., 2001) such that results were accepted as significant when FDR $\leq 0.006$. Adjusted p-values are indicated by "FDR" and unadjusted p-values are indicated by "p".

\subsubsection{Gene expression}

Cycle threshold (CT) values for each sample were averaged across replicates to obtain a mean CT value for each participant used in our analysis. All data was examined for outliers, and any replicates with a standard deviation greater than 0.25 were removed $(n=5)$, and the mean CT was re-calculated from the remaining data points (Biosystems, 2016). GE data were analyzed using the comparative CT method (Schmittgen and Livak, 2008), normalizing FKBP5 GE against the control gene TBP. Normalized data were analyzed using the student's t-test to compare expression levels according to exposure to $\mathrm{CM}$ and depression history. In this secondary analysis, results were accepted as significant if $\mathrm{p}<0.05$.

\subsubsection{Correlation analysis}

Pearson correlations were used to further explore the potential relationship between FKBP5 GE and $5 \mathrm{mC}$ at each of the four regions examined individually. Correlations were considered significant when $\mathrm{p}<0.05$. Tests were conducted using SPSS version 23.0.

\section{Results}

General descriptive statistics of the study sample are included in Table 1. Examination of the $5 \mathrm{mC}$ data revealed no extreme outliers. Participants with exposure to $\mathrm{CM}$ were not significantly different from those without CM exposure in terms of age, sex, self-reported race, genotype (rs1360780), PBMC count, or percent methylation (Table 1). There was a significant difference in CM score and depSS for participants with a history of CM exposure compared to those without such exposure (Table 1). Similarly, those with a history of depression had significantly different CM score and depSS compared to those with no reported history of depression (Table 1). A subset of the study participants $(\mathrm{N}=100)$ had available genotype information; of these, 16 participants were homozygous for the $\mathrm{T}$ risk allele (i.e. TT), 41 participants were heterozygous CT, and 43 were homozygous CC. Participant genotypes were in HardyWeinberg equilibrium ( $\mathrm{p}=0.248$ ). For SNP rs1360780, the major allele is $\mathrm{C}$ and the minor allele is $\mathrm{T}$. The minor allele frequency according to the 1000 genomes project for rs1360780 is 0.3273 (Genomes Project et al., 2015), while in this dataset the imputed minor allele frequency among participants is 0.365 .

\subsection{Mediation analysis}

\subsubsection{Upstream promoter}

All analyses of the promoter regions, both upstream and downstream, used the subsetted population of participants $(\mathrm{N}=72$,
$36 \mathrm{CM}$ cases). In the upstream promoter analyses, we first tested whether CM predicts depSS. CM was the sole significant predictor of depSS in this model $(\beta=4.78$ SE $1.11 \mathrm{p}<0.001$ FDR $=0.005$; Table S1a). The next linear regression model tested the relationship between $\mathrm{CM}$ and $5 \mathrm{mC}$; neither our primary predictor (i.e. $\mathrm{CM}$ ) nor any of the covariates were significantly associated with $5 \mathrm{mC}$ within this locus of the FKBP5 promoter region (Table S1b). The next regression model tested whether $5 \mathrm{mC}$ predicted depSS; no significant predictors or covariates were identified in this model (Table S1c). These results are summarized in Fig. 3A.

\subsubsection{Downstream promoter}

As with the upstream promoter region results, $\mathrm{CM}$ was the sole significant predictor of depSS in this model CM $(\beta=4.78$ SE 1.11 $\mathrm{p}<0.001$ FDR $=0.005$; Table S2a). Similarly, there was no significant relationship between $\mathrm{CM}$ or any of the covariates with $5 \mathrm{mC}$; and, no significant predictors were observed in the model examining whether $5 \mathrm{mC}$ predicted depSS (Tables $\mathrm{S} 2 \mathrm{~b}-\mathrm{c}$ ). These results are summarized in Fig. 3b.

\subsubsection{Intron 2}

All analyses of the intronic regions, both introns 2 and 7, drew on the full sample population ( $\mathrm{N}=112,56 \mathrm{CM}$ cases). In the first model, $\mathrm{CM}(\beta=4.47 \mathrm{SE} 1.44 \mathrm{p}=0.002 \mathrm{FDR}=0.006)$ significantly predicted depSS (Table S3a). In the next models, CM was not a significant predictor of FKBP5 Intron $25 \mathrm{mC}$ (Table S3b), nor did FKBP5 intron $25 \mathrm{mC}$ predict depSS (Table S3c). These results are summarized in Fig. 3C.

\subsubsection{Intron 7}

CM significantly predicted depSS $(\beta=4.47$ SE $1.44 \mathrm{p}=0.002$ $\mathrm{FDR}=0.006$; Table S4a). Similar to intron 2, CM was not a significant predictor of FKBP5 Intron $75 \mathrm{mC}$ (Table S4b). In the next regression model, intron $75 \mathrm{mC}$ and other covariates did not significantly predict depSS (Table S4c). Participant genotype was available for a subset of participants ( $\mathrm{N}=100,50 \mathrm{CM}$ cases); inclusion of participant genotype (rs1360780) as an interactor with CM (as previously shown (Klengel et al., 2013)), did not alter the reported non-significant findings (Fig. 3d; Supplementary Table 4).

\subsection{Gene expression of peripheral leukocytes}

FKBP5 GE was examined separately for CM and lifetime MDD to discern the potential impact of each exposure. No significant $(p>0.05)$ differences in relative GE levels were observed between the $\mathrm{CM}$ exposed cases compared to the non-exposed controls. However, when comparing MDD cases and controls, we observed a significant increase in relative GE level among those with MDD vs. controls ( $\mathrm{p}<0.001)$ (Fig. 4).

\subsection{Correlation of gene expression and DNA methylation}

The relationship between FKBP5 GE and $5 \mathrm{mC}$ at each of the four regions was examined to further elucidate the relationship. No significant ( $\mathrm{p}>0.05$ ) correlation between FKBP5 GE and 5mC was observed at any of the four loci examined: upstream promoter region $\mathrm{r}=0.036, \mathrm{p}=0.812$; downstream promoter region $\mathrm{r}=0.046$, $\mathrm{p}=0.759$; intron $2 \mathrm{r}=-0.063, \mathrm{p}=0.634$; intron $7 \mathrm{r}=0.091$, $\mathrm{p}=0.475$.

\section{Discussion}

Previous studies have highlighted the importance of early life environment in shaping mental health outcomes and have provided evidence that adverse early life experiences can impact $5 \mathrm{mC}$ 
Table 1

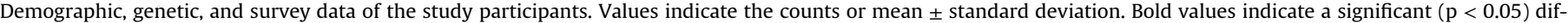
ference between groups.

\begin{tabular}{|c|c|c|c|c|c|c|c|}
\hline Measures & Full Study Sample & CM- & $\mathrm{CM}+$ & $\begin{array}{l}\mathrm{p} \text { value*, } \\
\text { t-test or } \mathrm{X}^{2}\end{array}$ & MDD- & MDD+ & $\begin{array}{l}\text { p value }{ }^{*}, \\
t \text {-test or } \mathrm{X}^{2}\end{array}$ \\
\hline \multicolumn{8}{|l|}{ Total $\mathrm{N}=112$} \\
\hline Age & $50.74 \pm 12.96$ & $50.11 \pm 13.14$ & $50.14 \pm 12.80$ & 0.988 & $51.50 \pm 13.32$ & $47.65 \pm 11.91$ & 0.131 \\
\hline Sex & & & & $\mathrm{X}^{2} 1$ & & & $X^{2} 0.257$ \\
\hline Male & 50 & 25 & 25 & & 30 & 20 & \\
\hline Female & 62 & 31 & 31 & & 42 & 20 & \\
\hline Race & & & & $X^{2} 0.697$ & & & $X^{2} 0.971$ \\
\hline European American & 18 & 8 & 10 & & 12 & 6 & \\
\hline African American & 86 & 43 & 43 & & 55 & 31 & \\
\hline Other & 8 & 5 & 3 & & 5 & 3 & \\
\hline rs1360780 & & & & $X^{2} 0.782$ & & & $X^{2} 0.385$ \\
\hline T Homozygous & 16 & 9 & 7 & & 12 & 4 & \\
\hline CT Heterozygous & 41 & 19 & 22 & & 23 & 18 & \\
\hline CC Homozygous & 43 & 22 & 21 & & 28 & 15 & \\
\hline Depression Symptom Severity & $8.28 \pm 7.82$ & $6.13 \pm 7.31$ & $10.47 \pm 7.78$ & 0.003 & $3.72 \pm 4.98$ & $16.38 \pm 4.80$ & $<0.001$ \\
\hline PBMC count (million cells) & $4.55 \pm 1.49$ & $4.50 \pm 1.31$ & $4.60 \pm 1.68$ & 0.719 & $4.46 \pm 1.42$ & $4.70 \pm 1.61$ & 0.432 \\
\hline CM Score & $6.12 \pm 5.43$ & $1.57 \pm 1.44$ & $10.66 \pm 3.93$ & $<0.001$ & $5.15 \pm 4.79$ & $7.85 \pm 6.11$ & 0.011 \\
\hline \multicolumn{8}{|l|}{ Percent DNA Methylation } \\
\hline Average Intron 2 & $76.40 \pm 6.19$ & $76.23 \pm 5.94$ & $76.61 \pm 6.50$ & 0.764 & $76.48 \pm 6.95$ & $76.30 \pm 4.45$ & 0.877 \\
\hline Average Intron 7 & $90.24 \pm 3.02$ & $89.98 \pm 3.14$ & $90.49 \pm 2.92$ & 0.397 & $90.01 \pm 2.76$ & $90.62 \pm 3.42$ & 0.326 \\
\hline \multicolumn{8}{|c|}{$\mathbf{N}=\mathbf{7 2}$} \\
\hline \multicolumn{8}{|l|}{ Percent DNA Methylation } \\
\hline Average Upstream Promoter & $4.64 \pm 1.67$ & $4.30 \pm 1.45$ & $4.75 \pm 1.87$ & 0.248 & & & \\
\hline Average Downstream Promoter & $2.80 \pm 0.93$ & $2.69 \pm 0.91$ & $2.90 \pm 0.95$ & 0.353 & & & \\
\hline Depression Symptom Severity & $3.72 \pm 4.98$ & $1.44 \pm 2.2$ & $6.06 \pm 5.91$ & $<0.001$ & & & \\
\hline CM Score & $5.15 \pm 4.79$ & $0.75 \pm 0.77$ & $9.56 \pm 2.48$ & $<0.001$ & & & \\
\hline
\end{tabular}

* Indicated p-values are derived from t-tests, unless denoted by $\mathrm{X}^{2}$, indicating it was derived by chi square test.

a

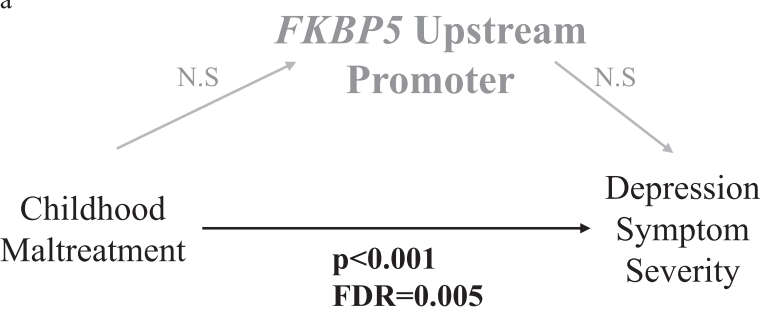

c

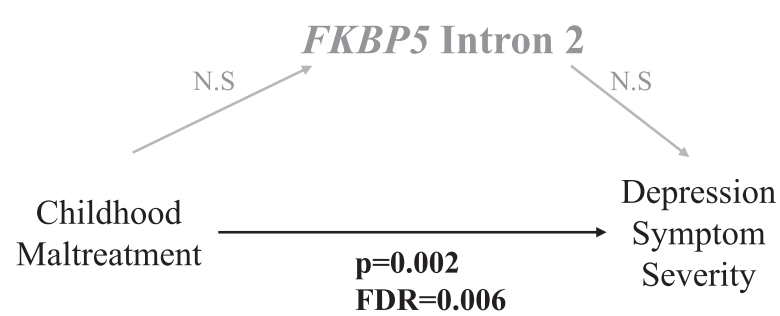

Childhood

Maltreatment

FDR $=0.005$ b

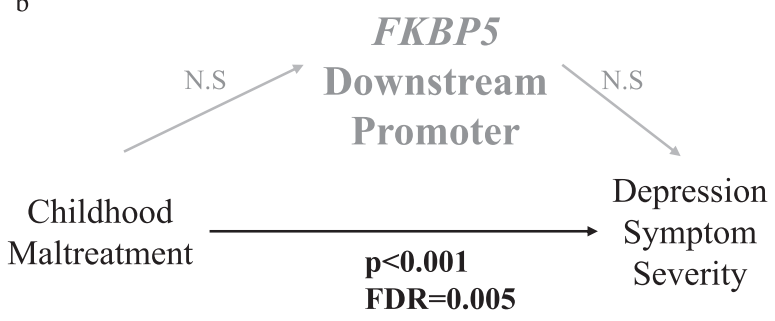

d

\section{Genotype}

(rs1360780)

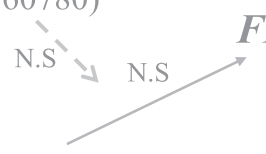

FKBP5 Intron 7

Childhood

Maltreatment

$\begin{array}{ll}\mathbf{p}=\mathbf{0 . 0 0 2} & \text { Symptom } \\ \mathbf{F D R}=\mathbf{0 . 0 0 6} & \text { Severity }\end{array}$

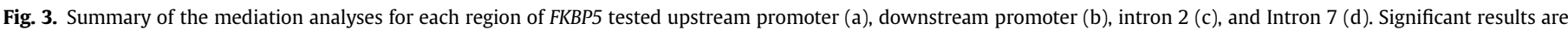
indicated by bold black text and denoted with both unadjusted p-values and FDR. Non-significant results are denoted by gray text and "N.S" for non-significant.

levels within HPA axis genes. Motivated by this work, we sought to test whether $5 \mathrm{mC}$ mediates the relationship between $\mathrm{CM}$ and depSS in an adult population. We examined four loci within FKBP5 (upstream promoter, downstream promoter, intron 2, and intron 7); the latter two loci have previously shown CM-associated $5 \mathrm{mC}$ differences. Our results suggest $5 \mathrm{mC}$ does not mediate the relationship between CM and depSS at any of the four FKBP5 loci examined. However, and confirming a large body of previous research in this area (Harkness and Monroe, 2002; Kessler et al., 1997; MacMillan et al., 2001; Molnar et al., 2001; Wanklyn et al., 2012), a significant effect of exposure to CM on depSS was observed. These results highlight the complex relationship between $\mathrm{CM}$ and depression onset, suggesting multiple factors may contribute to disease onset following exposure to CM. 


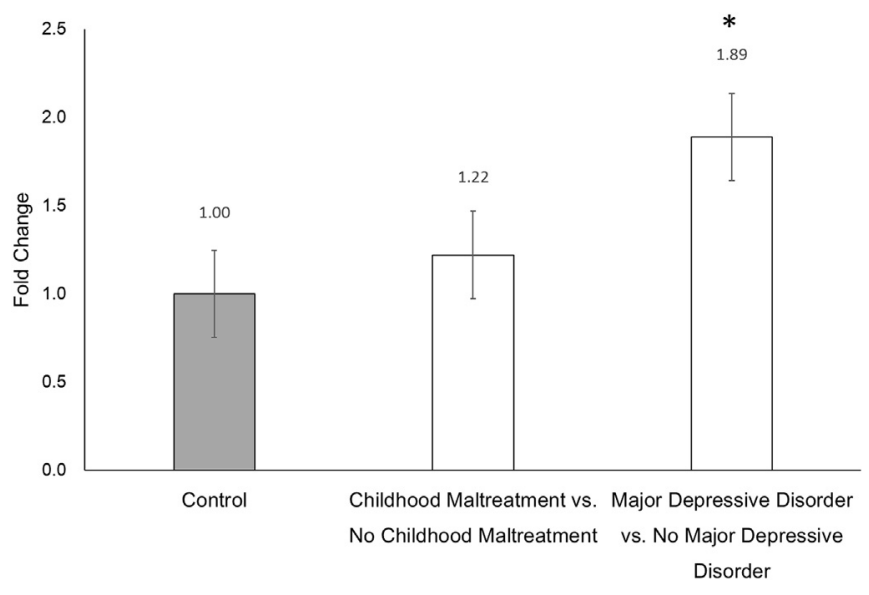

Fig. 4. FKBP5 gene expression levels among individuals with $(\mathrm{n}=35)$ vs. without $(\mathrm{n}=36)$ exposure to childhood maltreatment and participants with $(\mathrm{n}=25)$ vs. without $(\mathrm{n}=46)$ lifetime major depressive disorder history. T-tests showed a significant $(\mathrm{p}<0.001)$ increase in gene expression levels among major depressive disorder cases compared to controls. Error bars represent standard error and asterisk denotes significant $\mathrm{p}<0.05$

Further examination of the individual regression analyses of the larger mediation model revealed several surprising findings, the first of which is that we did not detect a significant effect of CM on FKBP5 5mC within intron 7 that has been reported in other studies (Klengel et al., 2013; Tyrka et al., 2015). However, similar to our findings for the promoter region, one previous study reported no significant effect of CM on FKBP5 promoter region $5 \mathrm{mC}$ (Klengel et al., 2013). The second surprising finding is that FKBP5 $5 \mathrm{mC}$ at any of the 4 loci were not significant predictors of depSS. Several studies have shown altered $5 \mathrm{mC}$ levels associated with depressive mood (Kahl et al., 2016; Nantharat et al., 2015; Oberlander et al., 2008; Tyrka et al., 2016). In addition, although the non-significant interaction between CM and genotype (rs1360780) at intron 7 in our data stands in contrast to previous work in adults (Klengel et al., 2013), this finding is consistent with a study in children (Tyrka et al., 2015). Further investigation is needed to elucidate the underlying mechanism between CM and FKBP5 5mC.

As a secondary analysis, we examined the potential significant functional effect of exposure to CM and lifetime MDD on FKBP5 GE levels. No effect of CM exposure was observed on FKBP5 GE levels. This finding is consistent with previous work that examined the biological correlates of PTSD in a longitudinal military cohort on FKBP5 GE and included CM as a covariate (van Zuiden et al., 2012). Examination of FKBP5 GE levels in relation to lifetime MDD, however, revealed a significant increase in expression between those with vs. without a history of the disorder. These findings are consistent with previous studies examining rodent GE of Fkbp5 in relation to chronic stress (Guidotti et al., 2013) or corticosterone exposure (Lee et al., 2010). One human study that compared FKBP5 GE in pre- and post-antidepressant treatment in healthy controls and depressed patients reported increased levels of FKBP5 GE at baseline for the depressed patients compared to the healthy controls (Cattaneo et al., 2013). Potential factors that may contribute to the increase of FKBP5 GE associated with lifetime MDD include cortisol, which may contribute to the observed association between FKBP5 GE and MDD history. Cortisol is released into the bloodstream from the adrenal glands when the HPA axis is activated during stressful events, actual or perceived. Cortisol, when bound to the GR increases transcription of FKBP5 via the ultra-short intracellular feedback loop. High cortisol levels and aberrant HPA axis regulation have been previously implicated in meta-analyses and multiple studies (Belvederi Murri et al., 2014; Dienes et al.,
2013; Stetler and Miller, 2011; Vreeburg et al., 2009), therefore, it is plausible that our study participants with lifetime MDD have altered cortisol sensitivity or HPA axis dysregulation, which contributes to the association between FKBP5 GE and lifetime MDD. Another potential mechanism contributing to this relationship is non-coding RNAs, which can modulate GE (Kaikkonen et al., 2011; Mattick and Makunin, 2006). Although our findings do not directly assess these possibilities, our results do provide additional evidence for the potential functional effect of depression on FKBP5 GE. The potential functional effect of increased GE levels associated with lifetime MDD may result in decreased sensitivity to cortisol, possibly conferring higher GR resistance (Lopez-Duran et al., 2015; Wei et al., 2015). In contrast, the non-significant effect of CM on $5 \mathrm{mC}$ at any of the four loci suggests there is no apparent functional effect of FKBP5 $5 \mathrm{mC}$ at these loci on FKBP5 GE levels, at least within the DNHS study population, as evidenced by the correlation analyses. In the present study we report no significant association between CM and $5 \mathrm{mC}$ in FKBP5, despite previous reports of an association between these two factors.

One potential reason for our findings, which do not replicate previous work, may pertain to the structure of our study. The population used in this study is drawn from a community-based, population-representative cohort, while the Klengel and Tyrka studies were predominantly comprised of participants from lower socioeconomic status. As such the level and types of trauma and/or contextual stressors experienced by both cases and controls may differ from our own population and impact results. Additionally, it is important to note the average participant age within each study. Similar to our study participants, the Klengel study is comprised of adults, whereas the Tyrka study is comprised of pre-school aged children. Within this study, the average age of DNHS participants was 50.74 years with maltreatment assessed prior to the ages of 11 and 18, resulting in an average of 32 years between exposure to $\mathrm{CM}$ and assessment of $5 \mathrm{mC}$. The Klengel study also used the CTQ to assess maltreatment prior to the age of 18 , however their population was on average younger than our DNHS participants, 41.46 years, resulting in an average of 22 years between exposure to $\mathrm{CM}$ and assessment of $5 \mathrm{mC}$. The Tyrka study, in contrast, included pre-school aged children and relied on child welfare records, rather than self-report, to assess CM. Given this information, the Klengel study is the most comparable to the current study in terms of $\mathrm{CM}$ assessment and age at $5 \mathrm{mC}$ assessment.

Another potential reason for non-replication may pertain to the difference in the definition of what constituted CM. Selection of CM cases within the Klengel study required histories of both sexual and physical abuse, whereas within this study, selection was based off of exposure levels defined by quartile, with no requirement regarding the type or number of maltreatment types. The Klengel study also reports a significant interaction of childhood trauma and FKBP5 SNP in predicting lifetime PTSD status and modified PTS symptom severity. Additionally, few studies have examined the impact of PTSD on 5mC in FKBP5 (Yehuda et al., 2013) and other HPA axis genes (Labonte et al., 2014; Yehuda et al., 2015b), therefore it is possible that co-morbid lifetime or current PTSD may be driving some of the reported significant findings in the earlier work. As a result, careful interpretation and comparison is required for our own findings as well as those reported by others.

There are study limitations that should be noted. First, the CM variable used in our study is a retrospective self-reported measure of abuses occurring before age 18. Early life experiences have been shown to be long lasting, with effects detected into adulthood, however self-reported measures may introduce recall bias. Previously, retrospective self-reports of $\mathrm{CM}$ from adults with documented cases were associated with underreporting of physical and sexual abuse (Widom and Morris, 1997; Widom and Shepard, 1996). Therefore, our measure of CM may well be an underestimate of 
previous abuse. Second, the use of self-reported measures may include heterogeneity and variability within the reported participant results for both measures of $\mathrm{CM}$ and depression, which may contribute to our reported null findings. Despite this possibility, the CM measure and a similar measure derived from the PHQ-9 for MDD were adequate to detect significant associations in our own previous work (Bustamante et al., 2016). Third, it is possible that our study was underpowered to detect the associations being explored within this work. Nevertheless, we do not believe this to be a likely explanation: the sample size used in this study, $\mathrm{N}=112$, is between the reported samples sizes for Klengel $(\mathrm{N}=76)$ and Tyrka $(\mathrm{N}=174)$ which both reported significant findings for FKBP5. Moreover, a post-hoc power calculation shows that the sample is adequately powered (86.5\%) to detect medium effects sizes as determined by $G^{*}$ Power (Faul et al., 2009). Fourth, we were unable to control for white blood cell subset within our study; however, we were able to include PBMC counts in each regression model. Notably, there was no significant difference in PBMC counts between participants with vs. without exposure to $\mathrm{CM}$ or with vs. without a history of depression. Lastly, we were unable to assess whether our findings were associated with differences in participant cortisol level, as cortisol was not collected within the parent study, the DNHS. Recent work suggests there is a genotype-dependent cortisol response to stress associated with FKBP5 SNP rs1360780 (Buchmann et al., 2014; Fujii et al., 2014; Hohne et al., 2015).

The DNHS is a non-clinical, community-based cohort of adults Detroit, Michigan residents. As such, this study provides us the opportunity to generalize our findings to the larger Detroit population. Second, our study is unique because antidepressant medication use is a covariate in our analyses; inclusion of this variable allows us to adjust for the potential effect of antidepressant medication on $5 \mathrm{mC}$ levels as previously reported (Melas et al., 2012; Perisic et al., 2010). Third, this study adds to the literature by examining the relationship among $\mathrm{CM}, 5 \mathrm{mC}$, depSS, and $\mathrm{GE}$, with additional analyses examining FKBP5 genotype and its potential interaction with CM. Lastly, we measured $5 \mathrm{mC}$ and GE in a subset of the same study participants, which allowed us to infer the potential impact of altered $5 \mathrm{mC}$ downstream on FKBP5 GE.

In conclusion, we found that $5 \mathrm{mC}$ in multiple genomic regions of FKBP5, a regulator of GR sensitivity, does not mediate the relationship between CM and depSS. We also found a significant increase in FKBP5 GE levels associated with lifetime MDD. We did not however, observe any significant difference in relative GE levels related to exposure to $\mathrm{CM}$. Taken together, these results suggest other, additional factors beyond $5 \mathrm{mC}$ likely mediate the relationship between CM and depression as detectable in blood. Future studies would benefit from the examination of participant cortisol levels to further assess the implications of altered FKBP5 GE within individuals with a history of MDD. Additionally, examination of $5 \mathrm{mC}$ within other regulatory regions of FKBP5 is critical to further understanding the role of FKBP5 in the context of CM and MDD.

\section{Conflicts of interest}

None.

\section{Acknowlegements}

This work was funded by NIH grants R01DA022720, R01DA022720-S1, and RC1MH088283.

\section{Appendix A. Supplementary data}

Supplementary data related to this article can be found at https://doi.org/10.1016/j.jpsychires.2017.09.016.

\section{References}

Afifi, T.O., Enns, M.W., Cox, B.J., Asmundson, G.J., Stein, M.B., Sareen, J., 2008. Population attributable fractions of psychiatric disorders and suicide ideation and attempts associated with adverse childhood experiences. Am. J. public health 98 (5), 946-952.

American Psychiatric, A, 1994. Diagnostic and Statistical Manual of Mental Disorders, fourth ed. American Psychiatric Association, Washintgon, DC.

Appel, K., Schwahn, C., Mahler, J., Schulz, A., Spitzer, C., Fenske, K., Stender, J., Barnow, S., John, U., Teumer, A., Biffar, R., Nauck, M., Volzke, H., Freyberger, H.J. Grabe, H.J. 2011. Moderation of adult depression by a polymorphism in the FKBP5 gene and childhood physical abuse in the general population. Neuropsychopharmacol. official Publ. Am. Coll. Neuropsychopharmacol. 36 (10) 1982-1991.

Baron, R.M., Kenny, D.A., 1986. The moderator-mediator variable distinction in social psychological research: conceptual, strategic, and statistical considerations. J. personality Soc. Psychol. 51 (6), 1173-1182.

Belvederi Murri, M., Pariante, C., Mondelli, V., Masotti, M., Atti, A.R., Mellacqua, Z., Antonioli, M., Ghio, L., Menchetti, M., Zanetidou, S., Innamorati, M., Amore, M., 2014. HPA axis and aging in depression: systematic review and meta-analysis. Psychoneuroendocrinology 41, 46-62.

Benjamini, Y., Drai, D., Elmer, G., Kafkafi, N., Golani, I., 2001. Controlling the false discovery rate in behavior genetics research. Behav. Brain Res. 125 (1-2), $279-284$.

Bernstein, Ahluvalia, Pogge, Handelsman, 1997. Validity of the childhood trauma Questionnaire in an adolescent psychiatric population. J. Am. Acad. Child. Adolesc. Psychiatry 36 (3), 340-348.

Binder, E.B., 2009. The role of FKBP5, a co-chaperone of the glucocorticoid receptor in the pathogenesis and therapy of affective and anxiety disorders. Psychoneuroendocrinology 34 (Suppl. 1), S186-S195.

Binder, E.B., Bradley, R.G., Liu, W., Epstein, M.P., Deveau, T.C., Mercer, K.B., Tang, Y. Gillespie, C.F., Heim, C.M., Nemeroff, C.B., Schwartz, A.C., Cubells, J.F., Ressler, K.J., 2008. Association of FKBP5 polymorphisms and childhood abuse with risk of posttraumatic stress disorder symptoms in adults. JAMA J. Am. Med. Assoc. 299 (11), 1291-1305.

Biosystems, A , 2016. Real-time PCR: Understanding Ct.

Buchmann, A.F., Holz, N., Boecker, R., Blomeyer, D., Rietschel, M., Witt, S.H. Schmidt, M.H., Esser, G., Banaschewski, T., Brandeis, D., Zimmermann, U.S. Laucht, M., 2014. Moderating role of FKBP5 genotype in the impact of childhood adversity on cortisol stress response during adulthood. Eur. Neuropsychopharmacol. J. Eur. Coll. Neuropsychopharmacol. 24 (6), 837-845.

Bustamante, A.C., Aiello, A.E., Galea, S., Ratanatharathorn, A., Noronha, C., Wildman, D.E., Uddin, M., 2016. Glucocorticoid receptor DNA methylation, childhood maltreatment and major depression. J. Affect. Disord. 206, 181-188.

Cattaneo, A., Gennarelli, M., Uher, R., Breen, G., Farmer, A., Aitchison, K.J., Craig, I.W. Anacker, C., Zunsztain, P.A., McGuffin, P., Pariante, C.M., 2013. Candidate genes expression profile associated with antidepressants response in the GENDEP study: differentiating between baseline 'predictors' and longitudinal 'targets'. Neuropsychopharmacol. official Publ. Am. Coll. Neuropsychopharmacol. 38 (3), 377-385.

Ceulemans, S., De Zutter, S., Heyrman, L., Norrback, K.F., Nordin, A., Nilsson, L.G., Adolfsson, R., Del-Favero, J., Claes, S., 2011. Evidence for the involvement of the glucocorticoid receptor gene in bipolar disorder in an isolated northern Swedish population. Bipolar Disord. 13 (7-8), 614-623.

Davies, T.H., Ning, Y.M., Sanchez, E.R., 2002. A new first step in activation of steroid receptors: hormone-induced switching of FKBP51 and FKBP52 immunophilins. Biol. Chem. 277 (7), 4597-4600.

Dienes, K.A., Hazel, N.A., Hammen, C.L., 2013. Cortisol secretion in depressed, and at-risk adults. Psychoneuroendocrinology 38 (6), 927-940.

Espejo, E. P. Hammen, C. L., Connolly, N.P. Brennan, P. A., Najman, J.M., Bor, W. 2007. Stress sensitization and adolescent depressive severity as a function of childhood adversity: a link to anxiety disorders. J. Abnorm. child Psychol. 35 (2) 287-299.

Faul, F., Erdfelder, E., Buchner, A., Lang, A.G., 2009. Statistical power analyses using G*Power 3.1: tests for correlation and regression analyses. Behav. Res. methods 41 (4), 1149-1160.

Fleige, S., Pfaffl, M.W., 2006. RNA integrity and the effect on the real-time qRT-PCR performance. Mol. Asp. Med. 27 (2-3), 126-139.

Fleige, S., Walf, V., Huch, S., Prgomet, C., Sehm, J., Pfaffl, M.W., 2006. Comparison of relative mRNA quantification models and the impact of RNA integrity in quantitative real-time RT-PCR. Biotechnol. Lett. 28 (19), 1601-1613.

Fujii, T., Hori, H., Ota, M., Hattori, K., Teraishi, T., Sasayama, D., Yamamoto, N. Higuchi, T., Kunugi, H., 2014. Effect of the common functional FKBP5 variant (rs1360780) on the hypothalamic-pituitary-adrenal axis and peripheral blood gene expression. Psychoneuroendocrinology 42, 89-97.

Genomes Project, C., Auton, A., Brooks, L.D., Durbin, R.M., Garrison, E.P., Kang, H.M., Korbel, J.O., Marchini, J.L., McCarthy, S., McVean, G.A., Abecasis, G.R., 2015. A global reference for human genetic variation. Nature 526 (7571), 68-74.

Goldmann, E., Aiello, A., Uddin, M., Delva, J., Koenen, K., Gant, L.M., Galea, S., 2011 Pervasive exposure to violence and posttraumatic stress disorder in a predominantly african american urban community: the Detroit neighborhood health study. J. Trauma. stress 24 (6), 747-751.

Guerry, J.D., Hastings, P.D., 2011. In search of HPA axis dysregulation in child and adolescent depression. Clin. child Fam. Psychol. Rev. 14 (2), 135-160. 
Guidotti, G., Calabrese, F., Anacker, C., Racagni, G., Pariante, C.M., Riva, M.A., 2013. Glucocorticoid receptor and FKBP5 expression is altered following exposure to chronic stress: modulation by antidepressant treatment. Neuropsychopharmacol. official Publ. Am. Coll. Neuropsychopharmacol. 38 (4) 616-627.

Harkness, K.L., Monroe, S.M., 2002. Childhood adversity and the endogenous versus nonendogenous distinction in women with major depression. Am. J. psychiatry 159 (3), 387-393.

Hohne, N., Poidinger, M., Merz, F., Pfister, H., Bruckl, T., Zimmermann, P., Uhr, M., Holsboer, F., Ising, M., 2015. FKBP5 genotype-dependent DNA methylation and mRNA regulation after psychosocial stress in remitted depression and healthy controls. Int. J. Neuropsychopharmacol./official Sci. J. Coll. Int. Neuropsychopharmacol. 18 (4).

Hussey, J.M., Chang, J.J., Kotch, J.B., 2006. Child maltreatment in the United States: prevalence, risk factors, and adolescent health consequences. Pediatrics 118 (3), $933-942$.

Jones, P.A., 2012. Functions of DNA methylation: islands, start sites, gene bodies and beyond. Nature reviews. Genetics 13 (7), 484-492.

Kahl, K.G., Georgi, K., Bleich, S., Muschler, M., Hillemacher, T., Hilfiker-Kleinert, D. Schweiger, U., Ding, X., Kotsiari, A., Frieling, H., 2016. Altered DNA methylation of glucose transporter 1 and glucose transporter 4 in patients with major depressive disorder. J. psychiatric Res. 76, 66-73.

Kaikkonen, M.U., Lam, M.T., Glass, C.K., 2011. Non-coding RNAs as regulators of gene expression and epigenetics. Cardiovasc. Res. 90 (3), 430-440.

Kessler, R.C., Davis, C.G., Kendler, K.S., 1997. Childhood adversity and adult psychiatric disorder in the US National Comorbidity Survey. Psychol. Med. 27 (5) $1101-1119$.

Keyes, K.M., McLaughlin, K.A., Koenen, K.C., Goldmann, E., Uddin, M., Galea, S., 2012 Child maltreatment increases sensitivity to adverse social contexts: neighborhood physical disorder and incident binge drinking in Detroit. Drug alcohol dependence 122 (1-2), 77-85.

Klengel, T., Mehta, D., Anacker, C., Rex-Haffner, M., Pruessner, J.C., Pariante, C.M. Pace, T.W.W., Mercer, K.B., Mayberg, H.S., Bradley, B., Nemeroff, C.B., Holsboer, F. Heim, C.M., Ressler, K.J., Rein, T., Binder, E.B., 2013. Allele-specific FKBP5 DNA demethylation mediates gene-childhood trauma interactions. Nat. Neurosci. 16 (1), 33-41.

Kroenke, K., Spitzer, R.L., Williams, J.B., 2001. The PHQ-9: validity of a brief depression severity measure. J. general Intern. Med. 16 (9), 606-613.

Labonte, B., Azoulay, N., Yerko, V., Turecki, G., Brunet, A., 2014. Epigenetic modulation of glucocorticoid receptors in posttraumatic stress disorder. Transl. psychiatry 4, e368.

Lee, R.S., Tamashiro, K.L., Yang, X., Purcell, R.H., Harvey, A., Willour, V.L., Huo, Y., Rongione, M., Wand, G.S., Potash, J.B., 2010. Chronic corticosterone exposure increases expression and decreases deoxyribonucleic acid methylation of Fkbp5 in mice. Endocrinology 151 (9), 4332-4343.

Lopez-Duran, N.L., McGinnis, E., Kuhlman, K., Geiss, E., Vargas, I., Mayer, S., 2015 HPA-axis stress reactivity in youth depression: evidence of impaired regulatory processes in depressed boys. Stress 18 (5), 545-553.

MacMillan, H.L., Fleming, J.E., Streiner, D.L., Lin, E., Boyle, M.H., Jamieson, E. Duku, E.K., Walsh, C.A., Wong, M.Y., Beardslee, W.R., 2001. Childhood abuse and lifetime psychopathology in a community sample. Am. J. psychiatry 158 (11) $1878-1883$.

Martin-Blanco, A., Ferrer, M., Soler, J., Salazar, J., Vega, D., Andion, O., Sanchez Mora, C., Arranz, M.J., Ribases, M., Feliu-Soler, A., Perez, V., Pascual, J.C., 2014 Association between methylation of the glucocorticoid receptor gene, childhood maltreatment, and clinical severity in borderline personality disorder. J. psychiatric Res. 57, 34-40.

Mattick, J.S., Makunin, I.V., 2006. Non-coding RNA. Human Molecular Genetics 15 Spec No 1, pp. R17-R29.

McGowan, P.O., Sasaki, A., D'Alessio, A.C., Dymov, S., Labonte, B., Szyf, M., Turecki, G. Meaney, M.J., 2009. Epigenetic regulation of the glucocorticoid receptor in human brain associates with childhood abuse Nat Neurosci. 12 (3), 342-348.

Melas, P.A., Rogdaki, M., Lennartsson, A., Bjork, K., Qi, H., Witasp, A., Werme, M. Wegener, G., Mathe, A.A., Svenningsson, P., Lavebratt, C., 2012. Antidepressant treatment is associated with epigenetic alterations in the promoter of P11 in genetic model of depression. Int. J. Neuropsychopharmacol./official Sci. J. Coll. Int. Neuropsychopharmacol. 15 (5), 669-679.

Molnar, B.E., Buka, S.L., Kessler, R.C., 2001. Child sexual abuse and subsequent psychopathology: results from the National Comorbidity Survey. Am. J. public health 91 (5), 753-760.

Na, K.S., Chang, H.S., Won, E., Han, K.M., Choi, S., Tae, W.S., Yoon, H.K., Kim, Y.K. Joe, S.H., Jung, I.K., Lee, M.S., Ham, B.J., 2014. Association between glucocorticoid receptor methylation and hippocampal subfields in major depressive disorder. PloS one 9 (1), e85425.

Nanni, V., Uher, R., Danese, A., 2012. Childhood maltreatment predicts unfavorable course of illness and treatment outcome in depression: a meta-analysis. Am. J. psychiatry 169 (2), 141-151.

Nantharat, M., Wanitchanon, T., Amesbutr, M., Tammachote, R., Praphanphoj, V., 2015. Glucocorticoid receptor gene (NR3C1) promoter is hypermethylated in Thai females with major depressive disorder. Genet. Mol. Res. GMR 14 (4), 19071-19079.

Nelson, J., Klumparendt, A., Doebler, P., Ehring, T., 2017. Childhood maltreatment and characteristics of adult depression: meta-analysis. Br. J. psychiatry J. Ment. Sci. 210 (2), 96-104.

Nemeroff, C.B., 2004. Neurobiological consequences of childhood trauma. J. Clin. psychiatry 65 (Suppl. 1), 18-28.

Non, A.L., Hollister, B.M., Humphreys, K.L., Childebayeva, A., Esteves, K., Zeanah, C.H. Fox, N.A., Nelson, C.A., Drury, S.S., 2016. DNA methylation at stress-related genes is associated with exposure to early life institutionalization. Am. J. Phys, Anthropol. 161 (1), 84-93.

Oberlander, T.F., Weinberg J., Papsdorf, M., Grunau, R., Misri, S., Devlin, A.M., 2008 Prenatal exposure to maternal depression, neonatal methylation of human glucocorticoid receptor gene (NR3C1) and infant cortisol stress responses. Epigenetics official J. DNA Methylation Soc. 3 (2), 97-106.

Perisic, T., Zimmermann, N., Kirmeier, T., Asmus, M., Tuorto, F., Uhr, M., Holsboer, F., Rein, T., Zschocke, J., 2010. Valproate and amitriptyline exert common and divergent influences on global and gene promoter-specific chromatin modifications in rat primary astrocytes. Neuropsychopharmacol. official Publ. Am. Coll. Neuropsychopharmacol. 35 (3), 792-805.

Purcell, S., Neale, B., Todd-Brown, K., Thomas, L., Ferreira, M.A., Bender, D., Maller, J., Sklar, P., de Bakker, P.I., Daly, M.J., Sham, P.C., 2007. PLINK: a tool set for wholegenome association and population-based linkage analyses. Am. J. Hum. Genet. 81 (3), 559-575.

Romens, S.E., McDonald, J., Svaren, J., Pollak, S.D., 2015. Associations between early life stress and gene methylation in children. Child. Dev. 86 (1), 303-309.

Schmittgen, T.D., Livak, K.J., 2008. Analyzing real-time PCR data by the comparative C(T) method. Nat. Protoc. 3 (6), 1101-1108

Stein, M.B., Walker, J.R., Anderson, G., Hazen, A.L., Ross, C.A., Eldridge, G., Forde, D.R. 1996. Childhood physical and sexual abuse in patients with anxiety disorders and in a community sample. Am. J. psychiatry 153 (2), 275-277.

Stetler, C., Miller, G.E., 2011. Depression and hypothalamic-pituitary-adrenal activation: a quantitative summary of four decades of research. Psychosom. Med. 73 (2), 114-126

Straus, M.A., 1979. Measuring intrafamily conflict and violence: the Conflict tactics(ct) scales. J. Marriage Fam. 41, 75-78.

Tyrka, A.R., Parade, S.H., Welch, E.S., Ridout, K.K., Price, L.H., Marsit, C., Philip, N.S., Carpenter, L.L., 2016. Methylation of the leukocyte glucocorticoid receptor gene promoter in adults: associations with early adversity and depressive, anxiety and substance-use disorders. Transl. psychiatry 6 (7), e848.

Tyrka, A.R., Price, L.H., Marsit, C., Walters, O.C., Carpenter, L.L., 2012. Childhood adversity and epigenetic modulation of the leukocyte glucocorticoid receptor: preliminary findings in healthy adults. PloS One 7 (1) e30148.

Tyrka, A.R., Ridout, K.K., Parade, S.H., Paquette, A., Marsit, C.J., Seifer, R., 2015. Childhood maltreatment and methylation of FK506 binding protein 5 gene (FKBP5). Dev. Psychopathol. 27 (4 Pt 2), 1637-1645.

Uddin, M., Aiello, A.E., Wildman, D.E., Koenen, K.C., Pawelec, G., de Los Santos, R., Goldmann, E., Galea, S., 2010. Epigenetic and immune function profiles associated with posttraumatic stress disorder. Proc. Natl. Acad. Sci. U. S. A. 107 (20), 9470-9475.

Uddin, M., Chang, S.C., Zhang, C., Ressler, K., Mercer, K.B., Galea, S., Keyes, K.M., McLaughlin, K.A., Wildman, D.E., Aiello, A.E., Koenen, K.C., 2013. Adcyap1r1 genotype, posttraumatic stress disorder, and depression among women exposed to childhood maltreatment. Depress, anxiety 30 (3), 251-258.

Uddin, M., Koenen, K.C., Aiello, A.E., Wildman, D.E., de los Santos, R., Galea, S., 2011. Epigenetic and inflammatory marker profiles associated with depression in a community-based epidemiologic sample. Psychol. Med. 41 (5), 997-1007.

van der Knaap, L.J., Riese, H., Hudziak, J.J., Verbiest, M.M., Verhulst, F.C., Oldehinkel, A.J., van Oort, F.V., 2014. Glucocorticoid receptor gene (NR3C1) methylation following stressful events between birth and adolescence. The TRAILS study. Transl. psychiatry 4, e381.

Van Voorhees, E., Scarpa, A., 2004. The effects of child maltreatment on the hypothalamic-pituitary-adrenal axis. Trauma Violence Abuse 5 (4), 333-352.

van Zuiden, M., Geuze, E., Willemen, H.L., Vermetten, E., Maas, M., Amarouchi, K. Kavelaars, A., Heijnen, C.J., 2012. Glucocorticoid receptor pathway components predict posttraumatic stress disorder symptom development: a prospective study. Biol. psychiatry 71 (4), 309-316.

Vreeburg, S.A., Hoogendijk, W.J., van Pelt, J., Derijk, R.H., Verhagen, J.C., van Dyck, R. Smit, J.H., Zitman, F.G., Penninx, B.W., 2009. Major depressive disorder and hypothalamic-pituitary-adrenal axis activity: results from a large cohort study. Archives general psychiatry 66 (6), 617-626.

Wanklyn, S.G. Day, D.M., Hart, T.A., Girard, T.A., 2012. Cumulative childhood maltreatment and depression among incarcerated youth: impulsivity and hopelessness as potential intervening variables. Child. Maltreat 17 (4), 306-317.

Weder, N., Zhang $\mathrm{H}$., Jensen, K., Yang B. Simen, A, Jackowski, A, Lipschitz, D. Douglas-Palumberi, H., Ge, M., Perepletchikova, F., O'Loughlin, K., Hudziak, J.J., Gelernter, J., Kaufman, J., 2014. Child abuse, depression, and methylation in genes involved with stress, neural plasticity, and brain circuitry. J. Am. Acad. Child Adolesc. Psychiatry 53 (4), 417-424 e415.

Wei, J., Sun, G., Zhao, L., Yang, X., Liu, X., Lin, D., Li, T., Ma, X., 2015. Analysis of hair cortisol level in first-episodic and recurrent female patients with depression compared to healthy controls. J. Affect. Disord. 175, 299-302.

Widom, C.S., Morris, S., 1997. Accuracy of adult recollections of childhood victimization: part 2. Childhood sexual abuse. Psychol. Assess. 9, 34-46.

Widom, C.S., Shepard, R.L., 1996. Accuracy of adult recollections of childhood victimization: part 1. Childhood physical abuse. Psychol. Assess. 8, 412-421.

Yang, X., Ewald, E.R., Huo, Y., Tamashiro, K.L., Salvatori, R., Sawa, A., Wand, G.S., Lee, R.S., 2012. Glucocorticoid-induced loss of DNA methylation in nonneuronal cells and potential involvement of DNMT1 in epigenetic regulation of Fkbp5. Biochem. biophysical Res. Commun. 420 (3), 570-575.

Yehuda, R., Daskalakis, N.P., Bierer, L.M., Bader, H.N., Klengel, T., Holsboer, F., 
Binder, E.B., 2015a. Holocaust exposure induced intergenerational effects on FKBP5 methylation. Biol. psychiatry 80 (5), 372-380.

Yehuda, R., Daskalakis, N.P., Desarnaud, F., Makotkine, I., Lehrner, A.L., Koch, E. Flory, J.D., Buxbaum, J.D., Meaney, M.J., Bierer, L.M., 2013. Epigenetic biomarkers as predictors and correlates of symptom improvement following psychotherapy in combat veterans with PTSD. Front. psychiatry 4, 118.

Yehuda, R., Flory, J.D., Bierer, L.M., Henn-Haase, C., Lehrner, A., Desarnaud, F, Makotkine, I., Daskalakis, N.P., Marmar, C.R., Meaney, M.J., 2015b. Lower methylation of glucocorticoid receptor gene promoter $1 \mathrm{~F}$ in peripheral blood o veterans with posttraumatic stress disorder. Biol. psychiatry 77 (4), 356-364. 